\title{
Gestión educativa en zonas de difícil acceso a partir del coronavirus: Provincia de Orellana, Ecuador
}

\author{
Educational management in hard-to-reach areas due to \\ the coronavirus: Orellana Province, Ecuador
}

\author{
MsC. Mónica del Rocío Aguilera Cruz ${ }^{1}$ \\ monica_c1989@hotmail.com \\ https://orcid.org/0000-0001-5983-1712 \\ MsC. María Verónica Chavesta Álava ${ }^{2}$ \\ mari-vero-2007@hotmail.com \\ https://orcid.org/0000-0002-5700-397X
}

Recibido: 1/9/2020, Aceptado: 1/11/2020

\begin{abstract}
RESUMEN
El cantón Aguarico se encuentra ubicado en la provincia de Orellana es considerado una zona rica en biodiversidad que busca crear espacios de inclusión y mejoramiento para su población razón por la cual la gestión educativa es un tema de gran impacto que buscan sus docentes y administrativos trabajar a fin de garantizar una educación de calidad, en el mes de marzo nuestro país sufrió una gran transformación la incertidumbre comenzó acechar a nuestros ecuatorianos esta realidad también se vivió en el cantón objeto de estudio el cual por ser una zona de difícil acceso se ha limitado en todos los sectores. La presente investigación tiene como objetivo general diagnosticar la Gestión educativa en zonas de difícil acceso a partir del coronavirus: Un estudio a las Unidades educativas del Cantón Aguarico - Provincia de Orellana, a fin de conocer mediante un diseño no experimental la realidad académica que enfrenta un cantón lleno de oportunidades y recursos turísticos pero pobre en inversión y atención académica. La metodología empleada presenta un diseño no experimental con alcance descriptivo y enfoque cuantitativo, la población objeto de estudio corresponde a 100 docentes que representan a 6 unidades educativas del cantón Aguarico, se aplicó un muestreo aleatorio simple que utiliza como técnica la encuesta como parte del proceso de recolección de datos.
\end{abstract}

Palabras clave: Gestión educativa, zona de difícil acceso, inclusión, calidad académica.

\section{ABSTRACT}

The Aguarico canton is located in the province of Orellana and is considered an area rich in biodiversity that seeks to create spaces for inclusion and improvement for its population, which is why educational management is a subject of great impact that its teachers and administrators seek to work at. In order to guarantee a quality

\footnotetext{
${ }^{1}$ Docente Unidad Educativa Valdivia, Ecuador

2 Docente Unidad Educativa "Unidad Popular", Ecuador
} 


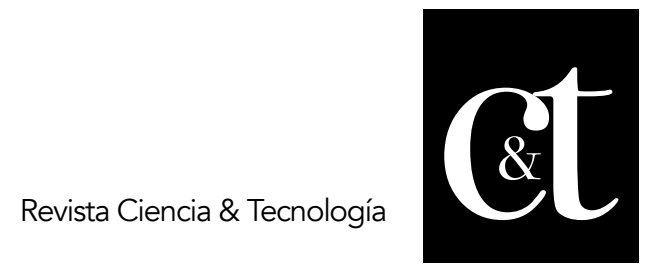

No. 29, 31 de enero de 2021

ISSN impreso: 1390 - 6321

ISSN online: 2661 - 6734

education, in the month of March our country underwent a great transformation, uncertainty began to haunt our Ecuadorians. This reality was also experienced in the canton under study, which, due to being an area of difficult access, has been limited in all sectors. The general objective of this research is to diagnose educational management in difficult-to-access areas from the coronavirus: A study of the educational units of the Cantón Aguarico - Orellana Province, in order to know through a non-experimental design, the academic reality faced by a canton full of tourism opportunities and resources but poor in investment and specific attention. The methodology used presents a non-experimental design with a descriptive scope and a quantitative approach, the population under study corresponds to 100 teachers representing 6 educational units of the Aguarico canton, a simple random sampling was applied that uses the survey as part of the process of data collection.

Keywords: Educational management, difficult access area, inclusion, academic quality.

\section{Introducción}

Durante los últimos meses la población a nivel nacional e internacional ha sufrido pérdidas irreparables que no solo han ocasionado daños a la economía, sino también a la educación, enfrentar la pandemia en el Ecuador ha sido una tarea muy difícil que el sistema educativo no ha sabido sobrellevar, como ecuatorianos no estábamos preparados para enfrentar una realidad tan compleja como la que estamos viviendo en la actualidad donde no solo se sufre las pérdidas humanas sino también el interés por la educación, esto sucede en el cantón Aguarico de la provincia de Orellana, una zona rica en biodiversidad, Aguarico se caracteriza por ser una zona turística y petrolera, este

cantón es la puerta para la investigación científica ya que aquí se encuentran áreas protegidas de gran renombre como son el parque Nacional Yasuní, y la reserva de Producción Faunística Cuyabeno, sin embargo, todos estos recursos no han sido aprovechados para mejorar la condición de vida de sus habitantes los cuales viven en una extrema pobreza.

La educación en esta zona también ha sufrido grandes pérdidas a tal punto que se ha considerado volver al modelo unidocente de la educación tradicional debido a la falta de inversión en el sector educativo, por otra parte las comunidades se veían obligadas a llevar a sus hijos a la Unidad Educativa Del Mileno Nuevo Rocafuerte institución creada con el objetivo de fusionar todas las Unidades Educativas de los alrededores, el objetivo fue brindar una educación digna para todos nuestros niños y jóvenes, esto se ha visto empañado por la falta de recursos económicos destinados al mantenimiento de la institución asi como también la falta de medios de transporte para el traslado de los estudiantes, esta situación empeoro con la presencia del coronavirus que en la actualidad nos tiene a todos en aislamiento a fin de mantener controlado el contagio y salvaguardar las vidas humanas. Las comunidades enfrentan graves problemas a nivel tecnológico la falta de recursos económicos sumado a la carencia de servicios básicos 


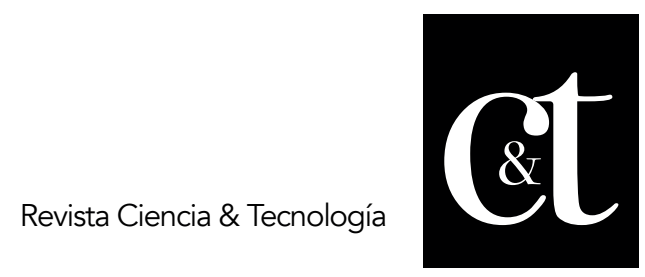

No. 29, 31 de enero de 2021

ISSN impreso: 1390 - 6321

ISSN online: 2661 - 6734

conlleva una lucha constante para los docentes que buscan que sus estudiantes continúen con el aprendizaje.

La Unesco por su parte, ha identificado grandes brechas en los resultados educativos, que se relacionan con una desigual distribución de los docentes, en general, y de los docentes mejor calificados, en particular, en desmedro de países y regiones con menores ingresos y de zonas rurales, las que suelen concentrar además a población indígena y migrante (UNESCO, 2020). Esta definición también la comparten las autoras quienes expresan que durante los últimos meses la gestión educativa llevada a cabo en las instituciones del cantón Aguarico han sufrido una gran transformación debido a que sus autoridades y docentes han buscado las mejores estrategias para evitar el retiro de estudiantes, una realidad a la que tuvieron que enfrentarse fue la educación en línea un modelo pedagógico nuevo y con altos índices de fracaso debido a la extrema pobreza en la que vive la población local, la educación en línea apareció como una estrategia del Ministerio de Educación a fin de garantizar el aprendizaje continuo en tiempo de pandemia.

Desde la postura científica Mariño (2016) refiere, la gestión educativa responde al "estudio de las nuevas dinámicas sociales y la tendencia hacia la descentralización de los sistemas educativos, genera que los docentes directivos asuman nuevos roles, tengan la capacidad para actuar de manera autónoma y tomar decisiones que le permitan acceder al mundo globalizado de la educación y de la sociedad en general" (p.12). Estos postulados expuestos por el autor constituyen una forma clara de representar las acciones de la comunidad educativa la cual debe crear espacios de armonía que conlleven a una educación de calidad, garantizando procesos coherentes y participativos. En el mismo contexto Asunción (2010) refiere "la gestión educativa es entendida como un proceso organizado y orientado a la optimización de procesos y proyectos internos de las instituciones, con el objetivo de perfeccionar los procedimientos pedagógicos, directivos, comunitarios y administrativos que en ella se movilizan" (p.11). En el mismo contexto Villavicencio, (2010) refiere "la gestión educativa adquiere un sentido más amplio que trasciende las fronteras del sistema educativo, al señalar como responsables del proceso educativo no solamente al Estado y a la institución escolar, sino al delegar responsabilidades a la sociedad y también al conceder alguna importancia a la educación no formal" (p.15).

Ríos (2010) considera que los directivos y docentes deben comprender que gerenciar una institución educativa no solamente implica la resolución de problemas o seguimiento de trámites administrativos, puesto que verdaderamente se debe procurar la aplicación de herramientas de gestión que permitan que una institución educativa (ya sea de nivel básico o superior) pueda desarrollar dos aspectos fundamentales como son la calidad educativa y gestión por logro de resultados; sin olvidar que el componente humano es el más importante, porque incide de manera directa y significativa en el logro de los aprendizajes de los alumnos (p.23). Por otra parte, Blanco (2010) sostiene, "cada institución construye y reconstruye su identidad; y gestiona procesos destinados al logro de la calidad sustentada en los valores, la equidad y la participación. Para llevarlos a cabo se necesitan modelos mentales compartidos, un pensamiento sistémico, la capacidad de liderazgo y el trabajo en equipo" (p.22). 


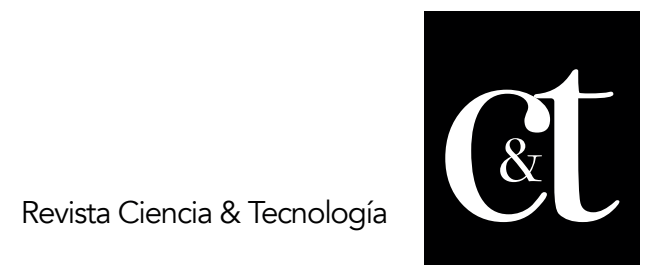

No. 29, 31 de enero de 2021

ISSN impreso: 1390 - 6321

ISSN online: 2661 - 6734

En el mismo orden de ideas Andrade (2010) refiere la "gestión se caracteriza por una visión amplia de las posibilidades reales de una organización para resolver alguna situación o alcanzar un fin determinado se define como el conjunto de acciones integradas para el logro de un objetivo a cierto plazo; es la acción principal de la administración y es un eslabón intermedio entre la planificación y los objetivos concretos que se pretenden alcanzar" (p.14).

Así mismo Montoya (2010) expone "la gestión educativa se constituye por la puesta en práctica de los principios generales de la gestión y de la educación en este sentido es una disciplina aplicada, es un campo de acción" (p.10). Para Biale (2010), la gestión educativa comprende "un conjunto de acciones que se realizan en una Institución Educativa de modo de facilitar la movilización de todos los elementos de su organización orientándolos hacia la concreción de las metas" (p.16).

Desde la concepción bibliográfica se determina que la gestión educativa debe ser considerada como una estrategia participativa que conlleve acciones de organización, definición que ha sido considerada por docentes y administrativos de las instituciones objeto de estudio quienes consideran que la educación en el cantón Aguarico no debe detenerse debido a que en muchos hogares otro problema a enfrentar es la violencia intrafamiliar razón por la cual los docentes deben trasladarse en canoas para llevar a cabo visitas domiciliarias y conocer de cerca la realidad de los estudiantes. Como parte de la gestión educativa que impulsa el Ministerio de Educación se encuentran las clases compartidas en televisión y radio, pero lastimosamente en este cantón estos recursos no son consideradas por la población educativa ya que estas comunidades carecen de energía eléctrica, razón por la cual la gestión educativa se encuentra orientada al acompañamiento pedagógico. El acompañamiento pedagógico buscar crear espacios de confianza y armonía con los estudiantes permitiendo que el docente conozca sus realidades.

Desde la concepción bibliografía el criterio expuesto por las autoras guardan una estrecha relación, debido a que la gestión educativa debe involucrar aspectos de acompañamiento y relación directa entre el profesor y el alumno a fin de garantizar una educación de calidad asi lo expresan también las autoras; Dra. María Elena Mellado, Mg. Carolina Villagra, Mg. Omar Aravena quienes sostienen.

El "antes" está relacionado con el diseño de la clase, donde acompañante y acompañado reflexionan acerca de la preparación de la enseñanza. Esta experiencia de planificación conjunta, constituye una instancia de desarrollo profesional, que puede llevarse a cabo en pequeños grupos de docentes organizados por asignaturas, departamentos, niveles o ciclos. El "durante" la clase, consiste en la implementación del diseño de la enseñanza para el aprendizaje, donde el acompañante asume un rol activo a través de la co-enseñanza, de manera de transformarse en un mediador coresponsable del desarrollo de habilidades, conocimientos y actitudes del estudiantado. El "después" debería comenzar con una conversación guiada a través de preguntas que gatillan la reflexión de la clase y permiten el análisis de las evidencias de desempeño de los estudiantes según los objetivos de aprendizaje.

Se espera que este proceso reflexivo constituya un hábito de la práctica y facilite al docente la autorregulación de su desempeño (Mellado, Villagra, \& Aravena, 2014). Diversas investigaciones han demostrado que la pobreza afecta el desarrollo evolutivo y social de los estudiantes, en gran parte los estudiantes presentan 


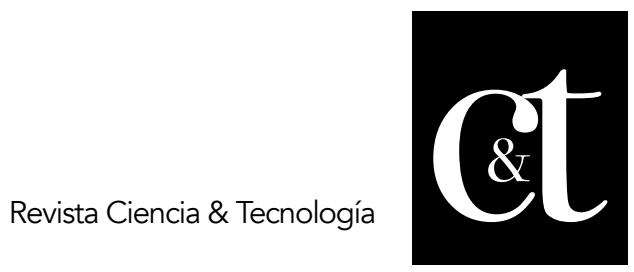

No. 29, 31 de enero de 2021

ISSN impreso: 1390 - 6321

ISSN online: 2661 - 6734

dificultades para el aprendizaje, en el aula, los estudiantes captan, razonan, reflexionan a diferentes ritmos, por eso los docentes no pueden manejar el aula como si todos estuviesen en las mismas condiciones, requiere identificar las necesidades y promover la motivación de los estudiantes hacia el aprendizaje (Cantillo \& Calabria, 2010). En el mismo orden de ideas Sandoval (2014) refiere, "la educación requiere un cambio substancial en los conceptos y conocimientos que incluya la capacidad de comprender lo que se ha aprendido, desarrollar capacidades, conocimientos e información en la vida diaria, aprendiendo a convivir en una sociedad más compleja" (p.13).

Siguiendo el orden de ideas se puede evidenciar que la gestión educativa responde a procesos de participación donde los docentes juegan un rol fundamental para el desarrollo del aprendizaje, el cantón Aguarico no solo es cuna de atractivos turístico sino también es un cantón lleno de oportunidades y espacios para la educación. Sus docentes cada día se esfuerzan por entregar a la sociedad jóvenes comprometidos con su comunidad y su entorno, la pandemia no ha detenido el proceso de enseñanza más bien a creado nuevos procesos para llevar a cabo una educación digna debido a que se han logrado acuerdos para la obtención de recursos destinados a zonas de difícil acceso a la vez se busca crear espacios que disminuyan los índices de pobreza. Bajo esta dimensión, la gestión educativa es entendida como un proceso organizado y orientado a la optimización de procesos y proyectos internos de las instituciones, con el objetivo de perfeccionar los procedimientos pedagógicos, directivos, comunitarios y administrativos que en ella se movilizan.

La institución es autónoma de definir sus criterios de gestión más adecuados y encaminados a responder con las necesidades educativas resultantes de la comunidad, más las exigencias legales de corte nacional e internacional (Rico, 2017). Asi mismo Mora (2010) refiere, "la gestión educativa se encarga de estudiar las diversas acciones, actividades, procesos y funcionamiento de los respectivos proyectos educativos institucionales particulares o específicos, y generales o globales, en ámbitos diversos del mundo educativo" (p.11). En tal sentido la gestión educativa es un proceso encaminado al fortalecimiento institucional que busca acercar a la comunidad educativa en el diseño de procesos educativos, en el cantón Aguarico los docentes y administrativos buscan fortalecer la Gestión académica mediante la aplicación de estrategias innovadoras que garanticen un aprendizaje de calidad permitiendo que los jóvenes guarden esperanzas de un mejor porvenir en su vida y fortaleciendo los estímulos académicos. La presente investigación tiene como objetivo general Diagnosticar la Gestión educativa en zonas de difícil acceso a partir del coronavirus: Un estudio a las Unidades educativas del Cantón Aguarico Provincia de Orellana, a fin de conocer mediante un diseño no experimental la realidad académica que enfrenta un cantón lleno de oportunidades y recursos turísticos pero pobre en inversión y atención académica.

\section{Metodología}

El estudio contempla un diseño no experimental con alcance descriptivo y enfoque cuantitativo, la población objeto de estudio corresponde a 100 docentes que representan a 6 unidades educativas del cantón Aguarico (Unidad Educativa Tiputini, Unidad Educativa Fronteras del Ecuador, Unidad Educativa del Milenio Nuevo Rocafuerte, Unidad Educativa Nampawe Onkyere Yatewe, Colegio San Francisco de 


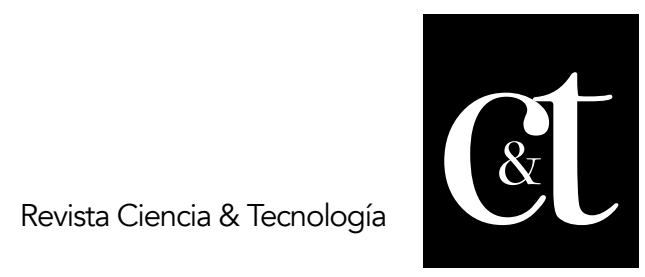

No. 29, 31 de enero de 2021

ISSN impreso: 1390 - 6321

ISSN online: 2661 - 6734

Asís, Pedro Camilo Tangoy), se aplicó un muestreo aleatorio simple debido a que al aplicar este tipo de muestreo se consideró que la unidad de análisis tiene la misma posibilidad de ser considerado en la toma de información, en la investigación la fórmula para calcular el tamaño de la población es la siguiente:

$$
\begin{aligned}
& N \sigma^{2} Z^{2} \\
& n=e^{2}\left(N-\overline{1)+\sigma^{2} l^{2}}\right.
\end{aligned}
$$

\section{Donde:}

$\mathrm{n}=$ el tamaño de la muestra.

$\mathrm{N}=$ tamaño de la población 100

$\sigma=$ Desviación estándar de la población que, generalmente cuando no se tiene su valor, suele utilizarse un valor constante de 0,5.

$Z=$ Valor obtenido mediante niveles de confianza. 1,96

e $=$ Límite aceptable de error muestral 0,05\%

\section{Reemplazando valores de la fórmula se tiene:}

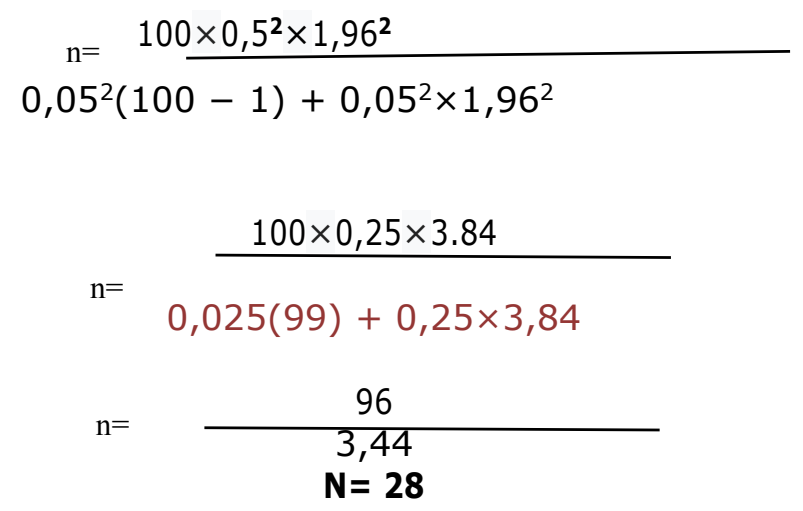

La técnica aplicada fue la encuesta, la cual utiliza como instrumento un cuestionario prediseñado de 13 preguntas a través de la plataforma Google Forms, las encuestas se aplicaron a 28 docentes de las instituciones educativas mencionadas. Los métodos aplicados en la investigación son el método deductivo el cual permitió realizar una revisión metódica de literatura bibliográfica otorgándole a la investigación un alto grado científico, el método inductivo se aplicó con el objetivo de conocer la problemática que enfrenta el cantón en relación a la gestión educativa.

\section{Resultados y discusión}

Como parte del estudio cuantitativo llevado a cabo en las unidades educativas del cantón Aguarico se ha logrado identificar que pese a que este cantón es una zona 


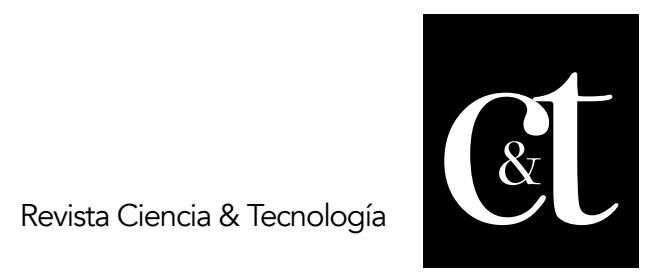

No. 29, 31 de enero de 2021

ISSN impreso: 1390 - 6321

ISSN online: 2661 - 6734

rica en biodiversidad los recursos destinados a educación aun no representan una mejora en el sistema educativo su población solicita de manera inmediata mayor atención, los jóvenes ven en el estudio una nueva esperanza muchos se encuentran sumergidos en problemas de alcoholismo debido a la falta de atención familiar de tal manera que en sus docentes encuentran un refugio así lo menciono la Lcda. Ana Lara quien mediante vía llamada telefónica expreso que como docentes conocer la realidad de cada estudiante durante esta pandemia ha sido un verdadero reto hemos tenido que dejar de lado nuestra parte profesional y trasladarnos hasta los hogares de los estudiantes para hacerlos sentir en confianza gastando nuestros recursos pero esto no importa debido a la necesidad de mejorar la educación en una zona alejada de nuestro país.

1. ¿Cuál es la gestión educativa aplicada en su institución con relación a las clases online?

28 respuestas

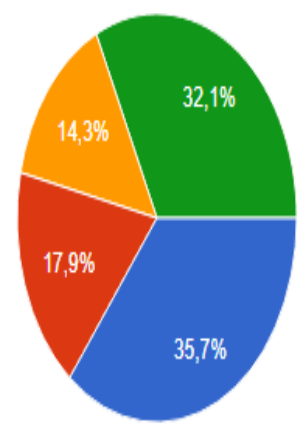

Capacitación docente

Diseño de plataforma académica

Capacitación a los padres de familia

Ninguna de las anteriores

\section{Figura 1. Resultado encuesta pregunta 1} Fuente: Estudio cuantitativo

Con respecto a la interrogante cual es la gestión educativa aplicada en su institución con relación a las clases online el $35,7 \%$ de los encuestados afirmo que la capacitación docente ha sido una de las mayores fortalezas de las autoridades con el afán de que los docentes puedan dar respuestas inmediatas a las necesidades educativas de cada alumno asi lo refleja el gráfico número uno que hace mención a esta interrogante, mientras que el $32,1 \%$ de encuestados respondió que ninguna de las opciones anteriores han sido socializadas a la planta docente. 


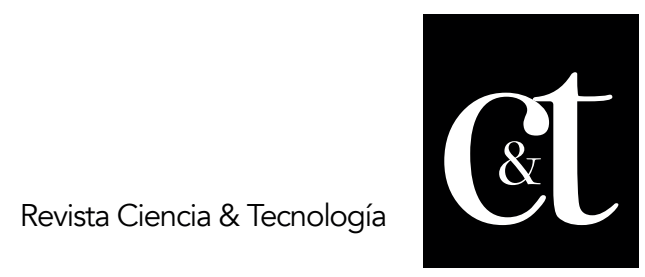

No. 29, 31 de enero de 2021

ISSN impreso: 1390 - 6321

ISSN online: 2661 - 6734

\begin{abstract}
3. ¿Cuál de los siguientes recursos educativos desarrollados para los estudiantes que no poseen conexión a internet se emplean con mayor frecuencia en su institución Educativa? 28 respuestas
\end{abstract}
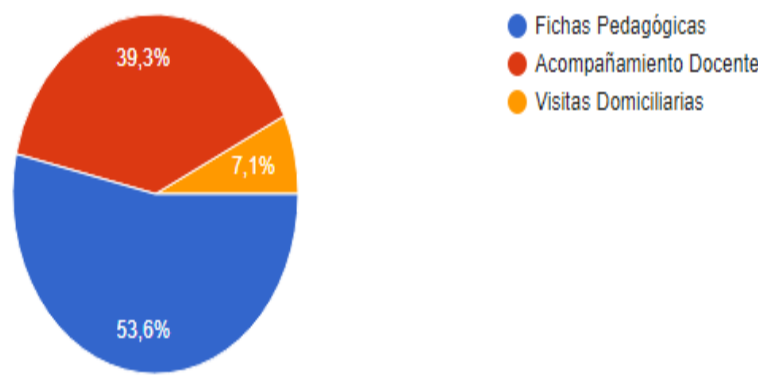
V Visitas Domiciliarias

\title{
Figura 2. Resultado encuesta pregunta 3 Fuente: Estudio cuantitativo
}

Las fichas pedagógicas entregadas a los estudiantes sin conexión reflejan una excelente gestión educativa en zonas de difícil acceso ya que estás han permitido acercar al docente con la realidad de los estudiantes en muchos hogares los padres de familia presentan un nivel de escolaridad muy bajo razón por la cual como parte de la estrategia pedagógica se llevan a sus casas las fichas pedagógicas como recursos necesarios para el aprendizaje continuo en la pregunta número 3 se evidencia que el $53,6 \%$ de encuestados considera que las fichas pedagógicas son los recursos empleados para estudiantes sin conexión mientras que otro 39,3\% reconoce al acompañamiento docente como otro de los recursos de mayor aceptación.

\section{5. ¿Considera usted que la educación se ha vulnerado en las comunidades?}

28 respuestas

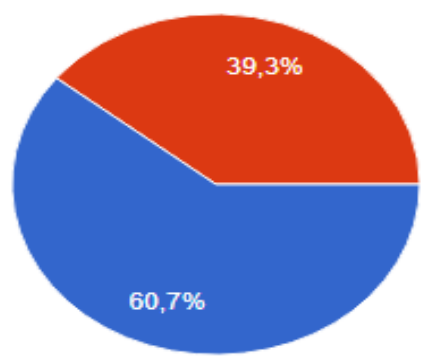

Figura 3. Resultado encuesta pregunta 5 Fuente: Estudio cuantitativo 


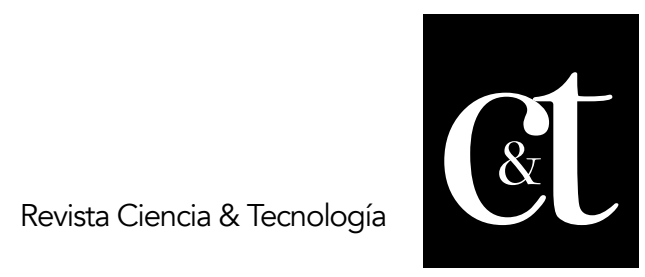

No. 29, 31 de enero de 2021

ISSN impreso: 1390 - 6321

ISSN online: 2661 - 6734

La pregunta numero 5 textualmente menciona que el $60,7 \%$ de encuestados reconocen que la educación si se ha vulnerado en las comunidades debido a la falta de recursos e intereses de inversión no solo para la infraestructura sino también para la contratación de planta docente al ser una zona de difícil acceso no se generan las garantías para los docentes y muchos de ellos tienden a renunciar en medio año lectivo debido a la falta de intereses por generar nuevas propuestas pedagógicas que mejoren la actividad educativa.

\section{8. ¿Cuántas veces a la semana los estudiantes se conectan a clases online? 25 respuestas}

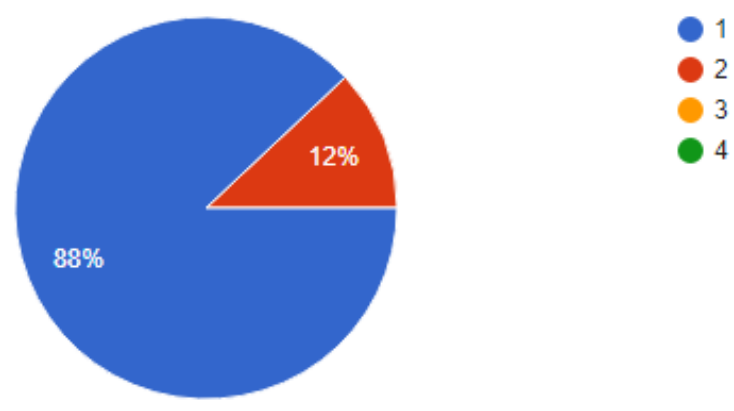

Figura 4. Resultado encuesta pregunta 8 Fuente: Estudio cuantitativo

Otro grave problema que enfrenta este cantón es la falta de conectividad al ser una zona de difícil acceso el internet es otro limitante como muestra la figura 4 el $88 \%$ de docentes encuestados menciona que solo 1 día a la semana se logran conectar los estudiantes lo que representa un verdadero reto debido a que muchos de ellos no cumplen las tareas, motivo por el cual como parte de la estrategia de gestión educativa se implementó un programa de clases dirigidas en casa las cuales tienen como principal actor a los docentes ellos realizan 2 veces por semana visitas domiciliarias en donde no solo brindan acompañamiento al estudiante sino al padre de familia el objetivo es promover que la educación no se detenga aquí se enseña al padre de familia a ser más colaborativo y a la vez participativo con la comunidad educativa. 


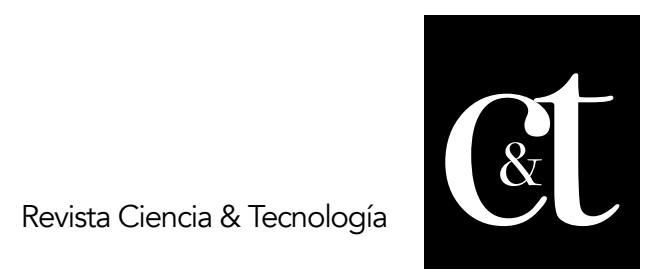

No. 29, 31 de enero de 2021

ISSN impreso: 1390 - 6321

ISSN online: 2661 - 6734

\section{9. ¿Existe en la institución casos de retiros voluntarios por falta de internet? \\ 27 respuestas}

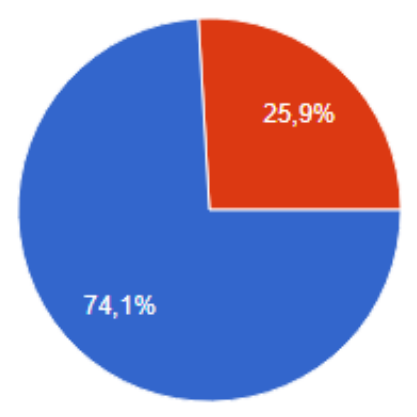

\section{Figura 5. Resultado encuesta pregunta 9} Fuente: Estudio cuantitativo

Lastimosamente en estas zonas los retiros voluntarios por falta de conectividad han sido otro reto que la comunidad educativa busca detener como muestra la figura 5 el $74,1 \%$ de encuestados mencionó que los padres han tomado la decisión de separar de la comunidad educativa a sus representados debido a la falta de recursos económicos que estos presentan total atención mostró el Distrito 22D03 Aguarico Educación en relación a esta dimensión razón por la cual como acción decidió crear un programa de cooperación entre el Gobierno Autónomo del cantón a fin de lograr recursos de financiamiento para el servicio de internet en la parroquia de Nuevo Rocafuerte.

\section{Propuesta de gestión para las Unidades educativas del Cantón Aguarico - Provincia de Orellana \\ En la gestión educativa en necesario que toda la comunidad educativa este en permanente comunicación, el proceso educativo debe observarse como un elemento integrador y participativo cuyo fin sea la calidad académica razón por la cual a continuación se presentan algunos elementos a considerar para el proceso de gestión educativa en zonas de difícil acceso.}

\section{Objetivos estratégicos}

- Mejorar el desempeño docente mediante la aplicación de programas de formación continua

Promover resultados en igualdad de condiciones a fin de consolidar en el cantón una educación inclusiva y de calidad.

\section{Políticas priorizadas}

Transparencia

Gestión

Descentralización e igualdad 


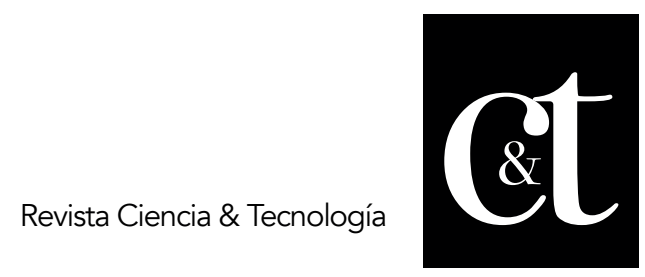

No. 29, 31 de enero de 2021

ISSN impreso: 1390 - 6321

ISSN online: 2661 - 6734

\section{Gestión}

Capacidad de las autoridades administrativas para desarrollar, concebir e integrar planes de acción en favor de la comunidad educativa buscando resaltar la visión y misión institucional académica.

\section{Gestión eficiente y transparente}

Promover la participación activa de la planta docente

Evaluar y monitorear recursos pedagógicos empleados por docentes de manera permanente

No hacer uso de los espacios institucionales para fines ajenos a la educación

Reconocer el código de ética institucional como un recurso de atención

Fomentar entre sus docentes espacios de diálogos y esparcimiento

\section{Dirección con liderazgo pedagógico}

Mejorar el aprendizaje de los alumnos

Crear espacios de inclusión

Establecer objetivos y metas en beneficio de la institución

Reconocer habilidades

Comunicar de manera oportuna los requerimientos institucionales entre sus docentes

\section{Características que debe presentar un director con liderazgo pedagógico Manejo emocional \\ Capacidad de escuchar \\ Manejo de grupos \\ Trabajo en equipo}

\section{Rediseñar la cultura educativa en zonas de difícil acceso}

Promover la formación de equipos pedagógicos

Involucrar a la familia en talleres educativos

Facilitar el trabajo pedagógico

\section{Conclusiones}

La gestión educativa responde a procesos organizados en donde toda la comunidad educativa juega un rol fundamental para el logro del éxito deseado, nuestro país no estaba preparado para una realidad tan difícil como la que vive en la actualidad muchos cantones sufren pérdidas irreparables, el cantón Aguarico no se encuentra alejado de esta realidad sus comunidades han logrado evidenciar las necesidades de invertir en educación a fin de lograr mejores oportunidades para sus jóvenes, a modo de conclusión se determina que la gestión educativa es un proceso que está siendo objeto de atención para la administración educativa a fin de solventar espacios inclusivos y el aprendizaje continuo.

El estudio determina que las zonas de difícil acceso representan espacios de vulneración de derechos motivo por el cual se ha determinado llevar a cabo programas de formación continua orientados a la planta docente a fin de solventar conflictos de retiro escolar. El cantón Aguarico es una zona rica en recursos los cuales deben ser orientados al mejoramiento de vida de la población local, la educación no puede ser objeto de programas pilotos en una zona como el lugar de estudio la educación se debe contemplar como un escenario de atención prioritaria donde 


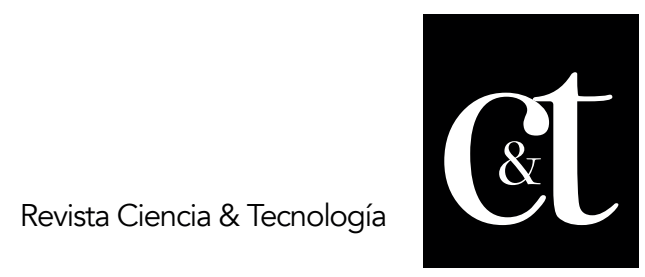

No. 29, 31 de enero de 2021

ISSN impreso: 1390 - 6321

ISSN online: 2661 - 6734

mejorar el nivel académico sea el elemento fundamental de la administración educativa, los docentes y administrativos de las unidades objeto de estudio orientan sus trabajos a la educación integradora pero esta debe requerir del apoyo de los entes gubernamentales y la familia nuestros jóvenes necesitan de administrativos que vean en ellos seres humanos comprometidos con la investigación y el aprendizaje científico.

\section{Referencias}

Andrade, M. (2010). Elementos y hechos educativos. Disciplinas educativas, 14.

Asunción, C. (2010). Gestión educativa como disciplina científica. Reflexiones educativas , 11 .

Biale, M. (2010). Elementos de la organización educativa. Hechos y sociedades de la educación, 16.

Blanco, S. (2010). Participación y equidad en la educación . Pedagógia y ciencia , 22.

Cantillo, B., \& Calabria, M. (2010). Prácticareflexiva.pro. Obtenido de https://practicareflexiva.pro/wpcontent/uploads/2019/03/Investigacio\%CC\%81n-sobreacompan\%CC\%83amiento-pedagogico.pdf

Mariño, P. (2016). La gestión educativa: Hacia la optimización de la formación docente en la educación superior en Colombia. Revista Shopia, 12.

Mellado, M., Villagra, C., \& Aravena, O. (2014). Liderazgo Educativo. Obtenido de https://liderazgoeducativo.udp.cl/columnas/acompanamiento-pedagogicocomo-estrategia-de-desarrollo-profesional-docente/

Montoya, S. (2010). Principios generales de la gestión. Modelos y hechos educativos, 10.

Mora, D. (2010). Objeto e importancia de la gestión educativa . Educación e investigación , 14.

Rico, A. (2017). Gstión educativa como un componente importante en la educación. Investigación y ciencia , 13.

Ríos, F. (2010). Instituciones educativas gerencias y análisis. Componente educativo, 23.

Sandoval, M. (2014). Reflexiones del aprendizaje. Aprendizaje significativo, 13.

UNESCO. (2020). repositorio. cepal.org. Obtenido de https://repositorio.cepal.org/bitstream/handle/11362/45904/1/S2000510_es.p df

Villavicencio, M. (2010). Gerencia educativa para lograr la transformación de las instituciones educativas. Investigación y análisis pedagógico, 15. 